\title{
An iterative ensemble Kalman filter for reservoir engineering applications
}

\author{
M. V. Krymskaya • R. G. Hanea • M. Verlaan
}

Received: 29 November 2007 / Accepted: 14 April 2008 / Published online: 14 June 2008

(C) The Author(s) 2008

\begin{abstract}
The study has been focused on examining the usage and the applicability of ensemble Kalman filtering techniques to the history matching procedures. The ensemble Kalman filter (EnKF) is often applied nowadays to solving such a problem. Meanwhile, traditional EnKF requires assumption of the distribution's normality. Besides, it is based on the linear update of the analysis equations. These facts may cause problems when filter is used in reservoir applications and result in sampling error. The situation becomes more problematic if the a priori information on the reservoir structure is poor and initial guess about the, e.g., permeability field is far from the actual one. The above circumstance explains a reason to perform some further research concerned with analyzing specific modification of the EnKF-based approach, namely, the iterative EnKF (IEnKF) scheme, which allows restarting the
\end{abstract}

M. V. Krymskaya $(\bowtie) \cdot$ M. Verlaan

Faculty of Electrical Engineering, Mathematics and Computer Science, Delft University of Technology, Mekelweg 4, 2628 CD Delft, The Netherlands e-mail: m.v.krymskaya@tudelft.nl

M. Verlaan

e-mail: verlaan@dutita2.twi.tudelft.nl

R. G. Hanea

TNO Built Environment and Geosciences,

Business Unit Geo Energy and Geo Information, TNO,

Princetonlaan 6, 3584 CB Utrecht, The Netherlands

e-mail: remus.hanea@tno.nl

R. G. Hanea

Faculty of Civil Engineering and Geosciences, Delft University of Technology,

Stevinweg 1, 2628 CN Delft, The Netherlands procedure with a new initial guess that is closer to the actual solution and, hence, requires less improvement by the algorithm while providing better estimation of the parameters. The paper presents some examples for which the IEnKF algorithm works better than traditional EnKF. The algorithms are compared while estimating the permeability field in relation to the twophase, two-dimensional fluid flow model.

Keywords Reservoir engineering • History matching • Permeability • Ensemble Kalman fitler •

Iterative ensemble Kalman filter

\section{Introduction}

The mathematical modeling approach to the analysis of reservoir performance has gained popularity throughout the years. However, the model can be used to forecast reservoir behavior only if it has been calibrated beforehand. The calibration stage, called "history matching" in the reservoir engineering context, aims at adjusting the parameters of the reservoir simulation model in such a way that the computed values of observable variables at individual wells are consistent with available measurements of those quantities. As the models become more complicated and larger scaled, there increases a need of automatic history matching techniques.

The main problem that has to be solved via automatic history matching is searching for the combination of reservoir parameters for which an error function (objective function) attains its minimum. This function represents a sum of squared differences between 
the observed reservoir performance and the results of simulation during the historical period [2]:

$f_{E}=\sum_{i=1}^{n_{\mathrm{par}}}\left[w_{i}\left(\mathbf{x}_{\mathrm{io}}-\mathbf{x}_{\mathrm{is}}\right)^{2}\right]$,

where $f_{E}$ denotes error function, $n_{\mathrm{par}}$ is the number of reservoir parameters, $w_{i}$ is the weighting coefficient, and $\mathbf{x}_{\text {io }}$ and $\mathbf{x}_{\text {is }}$ correspondingly refer to observed and simulated data that have to be matched. History matching is usually an ill-posed problem since there are more unknowns than constraints to resolve all the undetermined quantities. Then, the Gauss-Newton method applied to minimize cost function fails because the Hessian is ill-conditioned. Such a problem can be overcome by applying some regularization strategy (e.g., by use of the prior geostatistical model [8]).

There are several approaches to automatic history matching, which differ in the way they obtain the set of parameter values minimizing an objective function. The choice of minimization technique is mainly based on whether the error function has linear or nonlinear form.

However, most of these traditional history matching approaches are either limited to the small-scaled and simple reservoir models or inefficient in terms of computational costs. In general, these methods also perform the treatment of uncertainty via repeated history matching processes for various initial models, which results in even greater computational efforts. Moreover, traditional history matching does not allow continuous model updating. Namely, as the new data become available for being included into the match, the whole history matching process has to be repeated using all observed data. At the same time, the amount of deployed sensors for permanent monitoring of pressure, temperature or flow rates increases. This fact yields the increase of data output frequency and lightens up a problem of incorporating obtained data in the model as soon as it become available so that the model is always up-to-date.

The Kalman filtering techniques are known as the most popular methodology for assimilating the new measurements to continuously update the state of the system. Originally, the Kalman filter was developed for operating on the linear models, while nonlinearity requires using some further modifications, e.g., the extended Kalman filter. However, when the model is highly nonlinear or the scale of the space vector is too large, application of the extended Kalman filter also meets difficulties. These difficulties are overcome by applying the ensemble Kalman filtering (EnKF) algorithm based on the Monte-Carlo approach.
The great majority of the problems in reservoir engineering are highly nonlinear and characterized by a large number of variables; thus, the idea to use EnKF in reservoir simulation seems to be natural. In particular, it is presented in the publications [5, 10]. Other papers $[4,7]$ report the results of using the EnKF approach in history matching processes. They consider the application of EnKF to a PUNQ-S3 model. Although these studies clearly show that EnKF is successful in assimilating production data to update the initial reservoir model and its application allows reducing computational costs for history matching, there is still enough space for further investigation and improvement.

Specifically, the research described in [11] has shown that, for some nonlinear models, the EnKF does not provide completely acceptable characterizations of the uncertainties. The situation becomes more problematic if the a priori information on the reservoir structure is poor and the initial guess about the system state is far from the actual one. This leads to the idea of using improved EnKF modifications, namely, iterative EnKF (IEnKF) schemes, which allow improving the initial ensemble used for simulation and, hence, the resulting estimated state vector.

In this paper, we apply an iterative algorithm based on a sequential data assimilation scheme to estimating the permeability field in reservoir models. This algorithm is inspired by investigations of A.H. Jazwinski on an iterative extended Kalman filtering approach [6]. The implementation and the improvement of this method against the classical EnKF are presented with a $2 \mathrm{D}$ reservoir simulation oriented towards estimating the permeability field.

\section{The ensemble Kalman filter}

Kalman filtering is a powerful technique designed for solving data assimilation problems. This section presents the general idea of Kalman filtering in a manner similar to [9] and of ensemble Kalman filtering as given in [3]. Let us restrict ourselves to the case of the following linear system:

$\mathbf{x}_{k+1}=\mathbf{F}_{k} \mathbf{x}_{k}+\mathbf{B}_{k} \mathbf{u}_{k}+\mathbf{G}_{k} \mathbf{w}_{k}$,

$\mathbf{z}_{k}=\mathbf{M}_{k} \mathbf{x}_{k}+\mathbf{v}_{k}$,

where $\mathbf{F}_{k}, \mathbf{B}_{k}, \mathbf{G}_{k}, \mathbf{M}_{k}$ are matrices, $k$ is the time index, $\mathbf{x}_{k}$ denotes the state of the system, $\mathbf{u}_{k}$ is the system input, $\mathbf{z}_{k}$ is the vector of measurements, $\mathbf{w}_{k}$ is Gaussian white system noise process with zero mean and covariance matrix $\mathbf{Q}_{k}$, and $\mathbf{v}_{k}$ is Gaussian white measurement noise process with zero mean and covariance matrix 
$\mathbf{R}_{k}$. The initial state $\mathbf{x}_{0}$ is assumed to be Gaussian with mean $\mathbf{x}_{0}$ and covariance matrix $\mathbf{P}_{0}$. Moreover, processes $\mathbf{x}_{0}, \mathbf{w}_{k}$, and $\mathbf{v}_{k}$ are assumed to be independent from each other.

Vector $\mathbf{x}_{k}$, which contains information on the current system state, cannot be directly observed. However, it is possible to measure $\mathbf{z}_{k}$, which is some function of $\mathbf{x}_{k}$ affected by noise process $\mathbf{v}_{k}$. The idea is to use the available measurements $\mathbf{z}_{k}$ for estimating the state of the system $\mathbf{x}_{k}$.

To solve the filtering problem, Eqs. 2 and 3, we have to determine the probability density of the state $\mathbf{x}_{k}$ conditioned on the history of available measurements $\mathbf{z}_{1}, \ldots, \mathbf{z}_{l}$. It turns out that this conditional density function is Gaussian; hence, it can be characterized by means of a covariance matrix. However, for nonlinear model operator $\mathbf{F}_{k}$ (which is precisely the case of reservoir engineering applications), such conditional density function can be represented by the first two moments only approximately.

The EnKF has been examined and applied in a number of studies since it was first introduced by Geir Evensen in 1994. This filtering approach is relatively easy to implement and has affordable computational costs. The EnKF is based on a representation of the probability density of the state estimate at time $k$ by a finite number $N$ of randomly generated system states $\mathbf{x}_{k, i}, i=1, \ldots, N$. Equations to obtain the mean $\mathbf{x}_{k}$ and covariance matrix $\mathbf{P}_{k}$ of probability density of state $\mathbf{x}_{k}$ at time $k$ conditioned on the history of the measurements $\mathbf{z}_{1}, \ldots, \mathbf{z}_{l}$ via EnKF algorithm can be formulated as follows [3] (where superscripts $p$ and $u$ stand for predicted and updated system states):

- Initialization step:

$$
\mathbf{x}_{0, i}^{u} \sim \mathcal{N}\left(\mathbf{x}_{0}, \mathbf{P}_{0}\right), i=1, \ldots, N .
$$

- Forward step:

$$
\begin{aligned}
\mathbf{x}_{k, i}^{p} & =\mathbf{F}_{k} \mathbf{x}_{k-1, i}^{u}+\mathbf{B}_{k} \mathbf{u}_{k}+\mathbf{G}_{k} \mathbf{w}_{k, i}, \\
i & =1, \ldots, N, \\
\mathbf{x}_{k}^{p} & =\frac{1}{N} \sum_{i=1}^{N} \mathbf{x}_{k, i}^{p}, \\
\mathbf{L}_{k}^{p} & =\left[\mathbf{x}_{k, 1}^{p}-\mathbf{x}_{k}^{p}, \ldots, \mathbf{x}_{k, N}^{p}-\mathbf{x}_{k}^{p}\right]^{\mathrm{T}},
\end{aligned}
$$

where $\mathbf{L}_{k}^{p}$ defines an approximation of the covariance matrix $\mathbf{P}_{k}^{p}$ with rank $N$ :

$$
\mathbf{P}_{k}^{p}=\frac{1}{N-1} \mathbf{L}_{k}^{p} \mathbf{L}_{k}^{p \mathrm{~T}}
$$

- Assimilation step:

$$
\mathbf{K}_{k}=\mathbf{L}_{k}^{p} \mathbf{L}_{k}^{p \mathrm{~T}} \mathbf{M}_{k}^{\mathrm{T}} x *\left(\mathbf{M}_{k} \mathbf{L}_{k}^{p} \mathbf{L}_{k}^{p \mathrm{~T}} \mathbf{M}_{k}^{\mathrm{T}}+(N-1) \mathbf{R}_{k}\right)^{-1},
$$

where $\mathbf{K}_{k}$ is the so-called "Kalman gain" matrix determining the weights with which the measurements have to be incorporated into the model update outcome,

$$
\begin{aligned}
\mathbf{x}_{k, i}^{u} & =\mathbf{x}_{k, i}^{p}+\mathbf{K}_{k}\left(\mathbf{z}_{k}-\mathbf{M}_{k} \mathbf{x}_{k, i}^{p}+\mathbf{v}_{k, i}\right), \\
i & =1, \ldots, N, \\
\mathbf{x}_{k} & =\frac{1}{N} \sum_{i=1}^{N} \mathbf{x}_{k, i}^{u} .
\end{aligned}
$$

Note that Eq. 10 involves generating additional noise $\mathbf{v}_{k, i}$ while constructing the measurement set corresponding to the ensemble. This noise $\mathbf{v}_{k, i}$ has the same statistics as assumed for the observation errors. The perturbed measurements are necessary due to the fact that the absence of perturbation leads to the updated ensemble, which has too low variance and causes the divergence of the algorithm [1].

Actually, the forward model integration step within reservoir engineering framework can be performed by making a forward run of the reservoir simulator

$\mathbf{x}_{k, i}^{p}=\mathbf{F}\left(\mathbf{x}_{k-1, i}^{u}\right)$,

which can be developed separately and used as a black box in EnKF analysis.

It turns out that parameter estimation via EnKF is also possible. This can be done by constructing the following state vector:

$\mathbf{x}=\left[\begin{array}{l}\mathbf{m} \\ \mathbf{y}\end{array}\right]$,

where $\mathbf{y}$ consists of dynamic variables changing with time and $\mathbf{m}$ is a vector of static model parameters, which are constant in time and have to be estimated.

Now, the Kalman filter analysis is performed on the augmented state vector. The forward step of the algorithm results in updating only the dynamic variables with time and conserving the values of static parameters. However, at the assimilation step, the variables of both types are simultaneously updated, providing corrected estimations of the state vector and, hence, model parameters.

The model describing multiphase fluid flow in reservoir is highly nonlinear and the number of variables included into state space vector is very large, normally at least two per grid block. Although EnKF performs fairly good for this kind of problem, it sometimes fails 
to provide appropriate characterization of uncertainty. An example is given in [11] in relation to the case when the conditional pdf for the reservoir model is multimodal. Such a phenomenon results from the fact that model nonlinearity destroys the normality of a prior and a posterior distributions within Kalman filtering analysis.

Moreover, the task of obtaining accurate estimation of the state vector becomes harder if the a priori information about the reservoir structure is poor and the initial guess about, e.g., permeability field is far from actual one. The Kalman filter techniques are designed in such a way that initial conditions tend to be forgotten by the filter algorithm as more data are assimilated [9]. However, it is important for reservoir engineering cases that reasonable estimations of the model parameters are obtained based on the data collected before the water breakthrough events. In turn, these data may not be enough to improve a poor initial guess about the field structure. In such a situation, for some applications, the idea of iterating filter globally can be of help since it allows restarting the procedure with a new initial guess that is closer to the actual solution and, hence, requires less improvement by the algorithm.

We are going to consider the history matching via EnKF algorithm as the starting point for further investigations. We continue with some introduction into alternative EnKF techniques.

\section{Iterative Kalman filtering}

The current section presents the ideas of Kalman filtering algorithms that, in our opinion, can be alternatively applied to solving the history matching problem. Iterative forms of the Kalman filter are not completely new within the scope of reservoir engineering applications. These methods aim at obtaining any ensemble that provides improving the representation of the state distribution. There exist several approaches in petroleum engineering literature, e.g., the ad-hoc confirming EnKF method proposed by [10].

We would like to exploit the idea of Jazwinski to iterate the filter globally [6]. Although it was originally suggested to iterate the extended Kalman filter, we modify the approach for the case of parameter estimation via EnKF technique.

The algorithm looks as follows. Incorporating all available data via EnKF starting with $\mathbf{x}_{0}$ and $\mathbf{P}_{0}$, we obtain the estimated values of $\mathbf{x}_{t_{\text {end }}}$ and $\mathbf{P}_{t_{\text {end }}}$, where $t_{\text {end }}$ denotes the end time point of data assimilation period. If the number of available measurements is sufficiently large, we can expect that the estimated model parameter value $\mathbf{m}_{t_{\text {end }}}$ is closer to the "true" one than initial $\mathbf{m}_{0}$. The estimated model parameter $\mathbf{m}_{t_{\text {end }}}$ now replaces $\mathbf{m}_{0}$ and becomes a new initial guess for the next global iteration, which is done by rerunning the EnKF based on the same bunch of observations. Afterwards, this procedure can be repeated until no sufficient change in estimated model parameter is obtained. Note that, when rerunning the filter, we change only the mean estimator of initial guess about the model parameter and not the statistics $\mathbf{y}_{0}$ and $\mathbf{P}_{0}$. This yields that, in the case of Gaussian initial ensemble, the new initial distribution can be generated by resampling with updated mean $\left[\mathbf{m}_{t_{\text {end }}}, \mathbf{y}_{0}\right]^{\mathrm{T}}$ and the same initial covariance $\mathbf{P}_{0}$. The flowchart of such an IEnKF is presented in Fig. 1, where the dashed blocks correspond to the steps that actually are the parts of the EnKF algorithm.

Although the more educated choice of initial guess naturally should result in better estimation, there is no guarantee that iteration will converge. Thus, the plan is to investigate the features of the above IEnKF technique and to check whether it indeed allows improving the state vector estimations. The next section outlines the settings of the experiment used to test EnKF and IEnKF performances.

Fig. 1 IEnKF algorithm flowchart

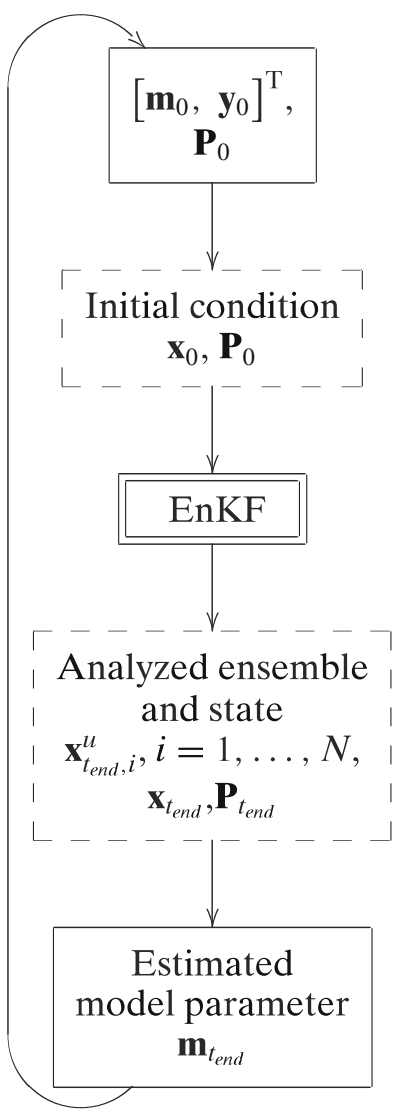




\section{Experimental environment}

The study is accomplished on the basis of a twodimensional, two-phase fluid flow model. The model implementation is provided by the forward reservoir simulator used as a black box to perform the time update in filtering algorithm.

The model is applied to a two-dimensional squared petroleum reservoir with a size of $700 \times 700 \mathrm{~m}$ equipped with uniform cartesian grid consisting of 21 grid cells in each direction. The reservoir is taken to be $2 \mathrm{~m}$ in height; however, we assume that all quantities are vertically homogeneous, which allows considering fluid flow processes only in two dimensions.

We consider the water flooding stage of the recovery process, which is performed through the exploitation of the injection well located at the center of the reservoir and four production wells established at the corners of the field. The injection well is constrained by a prescribed injection rate of $0.002 \mathrm{~m}^{3} / \mathrm{s}$ and production wells-by bottom hole pressure of $2.5 \times 10^{7} \mathrm{~Pa}$.

\subsection{State space representation}

In the case of reservoir engineering applications, the state vector normally consists of vectors of pressures (p) and saturations (s) corresponding to each grid block. To perform parameter estimation, we have to include the parameter of interest into the space vector. The study is focused on estimating the permeability field. It turns out that the natural logarithm of permeability is normally distributed, hence, we would like to augment the state vector by the vector of log-permeability $(\log \mathbf{k})$ corresponding to each grid cell. Moreover, while operating on a field, one can measure the following parameters at the wells: bottom hole pressures ( $\left.\mathbf{p}_{\text {well }}\right)$, oil $\left(\mathbf{q}_{\text {well }, o}\right)$, and water $\left(\mathbf{q}_{\text {well }, w}\right)$ flow rates. We can also include into available measurements the pressure and saturation quantities at the wells. Finally, the mega state space vector takes the following form:

$\mathbf{x}=\left[\begin{array}{c}\log \mathbf{k} \\ \mathbf{p} \\ \mathbf{s} \\ \mathbf{p}_{\text {well }} \\ \mathbf{q}_{\text {well }, o} \\ \mathbf{q}_{\text {well }, w}\end{array}\right]$.

The model parameter is considered as static, i.e., timeinvariant. Meanwhile, the value of the static parameter is corrected within the data assimilation step.

Note that the state vector consists of 441 permeability values, 441 pressure values, 441 water saturation values, 1 observed bottom hole pressure at the injection well, 4 observed oil flow rates, and 4 observed water flow rates at the production wells, or simply, $\mathbf{x} \in \mathbf{R}^{1332}$.

The relation Eq. 3 between the model variables and the measurements for our example is assumed to be given through trivial measurement matrix $\mathbf{M} \in \mathbf{R}^{19 \times 1332}$ with only zeros and ones as its elements, arranged in the block form:

$\mathbf{M}=\left[\begin{array}{llll}\mathbf{0} & \mathbf{M}^{1} & \mathbf{0} & \mathbf{0} \\ \mathbf{0} & \mathbf{0} & \mathbf{M}^{1} & \mathbf{0} \\ \mathbf{0} & \mathbf{0} & \mathbf{0} & \mathbf{M}^{2}\end{array}\right]$

with blocks $\mathbf{M}^{1} \in \mathbf{R}^{5 \times 441}$ and $\mathbf{M}^{2} \in \mathbf{R}^{9 \times 9}$ of the following form:

- Elements of matrix $\mathbf{M}^{1}$ corresponding to the observations at the well grid blocks [i.e., elements indexed as $(1,1),(2,21),(3,221),(4,421)$, and $(5,421)$ are set to one, the rest of the matrix if filled in with zeros].

- $\mathbf{M}^{2}$ is, in fact, an identity matrix.

Let us note that, although the measurement operator has a linear form, the actual relation between the model state vector and observable variables is nonlinear. The derived notation only shifts the source of nonlinearity and does not vanish its effects.

We consider the model Eq. 12 as being perfect, which might seem to be not very realistic. However, such an assumption specifies better environment for investigating a particular IEnKF method. We expect that, in the case when the ensemble spread is not influenced by model noise, the iterative techniques have to demonstrate their specific features. On the contrary, the values of observable variables are assumed to be imprecise. To initialize the filter, one needs generating initial ensembles of only static and dynamic variables because there is no production data available at the starting time.

Since the reservoir is typically in a state of equilibrium at the time when production starts, the initial dynamic variables (i.e., initial pressures and water saturations corresponding to each grid block) are assumed to be perfectly known (without uncertainty). Therefore, they are the same for each ensemble member and equal to the initial condition of the "true" model (i.e., $p=$ $3 * 10^{7} \mathrm{~Pa}$ and $S_{w}=0.2$ ).

Thus, at the initial moment, the only permutations contained in the initial ensemble are caused by initial ensemble of permeability models. Within the study, we are going to use the initial permeability ensemble consisting of 999 members. The ensemble mean and 


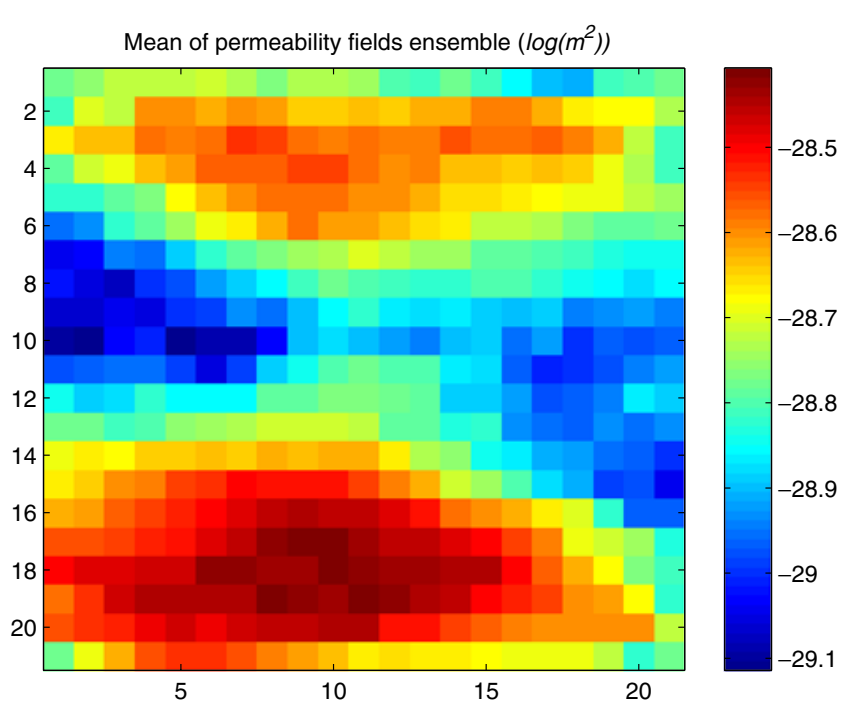

(a)

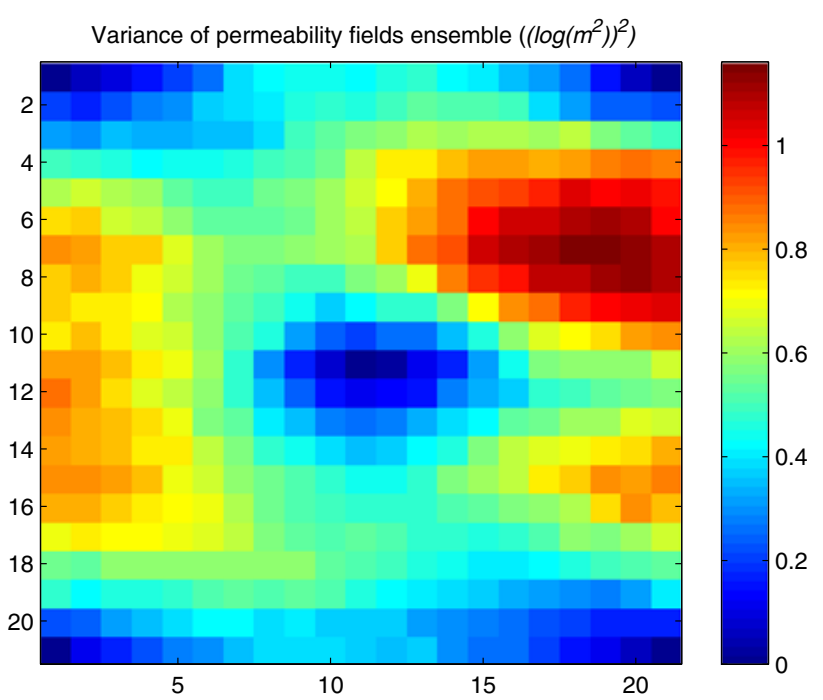

(b)

Fig. 2 Mean (a) and variance (b) of permeability fields ensemble

variance are visualized in Fig. 2, where the top picture corresponds to the ensemble mean and the bottom image to the variance, respectively.

\subsection{Synthetic measurements generation}

To test the performance of EnKF algorithms, we are going to do a so-called twin experiment. It requires that the "true" values of observable variables are generated synthetically by a preliminary run of the model itself and the noisy observations are then created by permutating the true values with the measurement error noise. This procedure ensures that the model is indeed able to match the data. Thereafter, the synthetic data are used in the assimilation experiments. The implementation of our in-house simulator provides the "true" permeability field (see Fig. 3), which originates from the training image of meandering channels.

Now it is possible to generate synthetic data initializing the simulator with true permeability field, grid block pressures $p=3 * 10^{7} \mathrm{~Pa}$, and water saturations $S_{w}=0.2$. The error in each observable variable is taken to be $5 \%$ of its actual scale. The same covariance matrix is then used to represent the measurements noise within data assimilation analysis.

\subsection{Measures of filter performance}

We quantify the quality of estimating a true permeability field in terms of the following space averaged root mean square (RMS) errors at time $k$ :

$\operatorname{RMS}\left((\log \mathbf{k})_{k}\right)=\sqrt{\frac{\left\|(\log \mathbf{k})_{k}-(\log \mathbf{k})^{\text {true }}\right\|_{2}^{2}}{\operatorname{dim}(\log \mathbf{k})}}$,

or

$\operatorname{RMS}\left((\log \mathbf{k})_{k, i}\right)=\sqrt{\frac{\left\|(\log \mathbf{k})_{k, i}-(\log \mathbf{k})^{\text {true }}\right\|_{2}^{2}}{\operatorname{dim}(\log \mathbf{k})}}$,

where $\operatorname{dim}(\log \mathbf{k})$ states for the size of vector of estimated parameters (i.e., $\operatorname{dim}(\log \mathbf{k})=441$ in our study), $(\log \mathbf{k})_{k}$ is the estimated vector of $\log$-permeability after the $k^{\text {th }}$ assimilation step, vector $(\log \mathbf{k})_{k, i}$ denotes the $i^{\text {th }}$ updated ensemble member after the $k^{\text {th }}$ assimilation step, and vector $(\log \mathbf{k})^{\text {true }}$ represents the true permeability field.

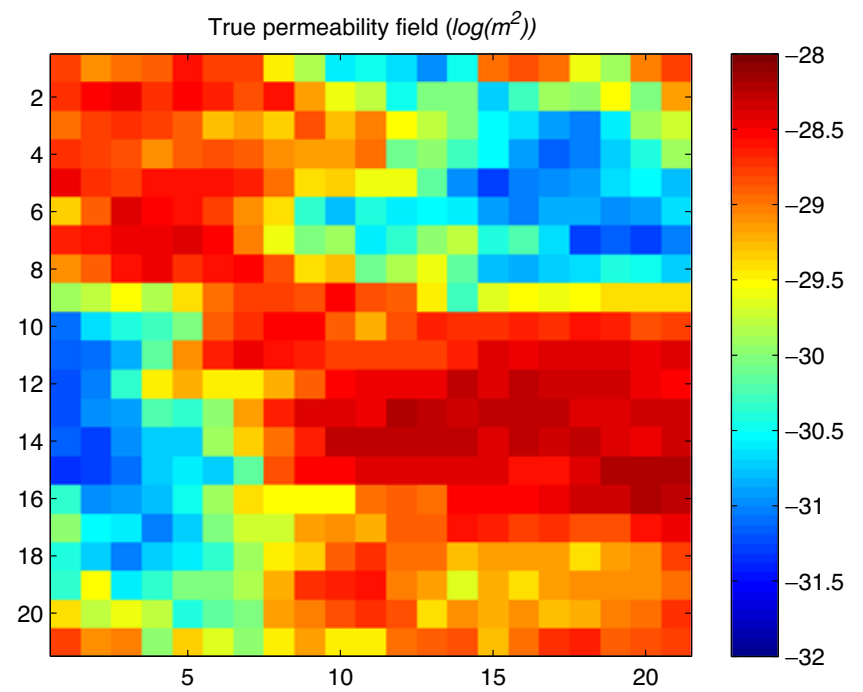

Fig. 3 "True" permeability field 


\section{Results and discussion}

A sequence of simulations has been accomplished to test the performance of EnKF and IEnKF assimilation algorithms in the framework of estimating the model parameters for a two-phase two-dimensional fluid flow model. We proceed by describing particular instances and discussing the obtained results.

The study of each particular algorithm includes solving the history matching problem and obtaining the estimate of model static parameter (i.e., permeability). The filter analysis is done from time $t_{0}=0$ days till $t_{\text {end }}=510$ days, which ensures that the water breakthrough event occurs in none of the production wells.

The use of classical EnKF in reservoir engineering framework meets an important obstacle concerned with obtaining physically unreasonable values of the state variables. It originates from performing data assimilation on the state vector without any constraint coming from the physical nature of the parameters. Hence, the updated dynamic variables may become unfeasible and inconsistent with estimated static variables. The authors of [10] proposed to include one additional socalled "confirming" step into the EnKF algorithm in order to ensure that the updated state is physically plausible. The idea of the confirmation step is the following: Starting at time moment $k-1$, we, at first, perform a forward simulator run up to time $k$ and then a data assimilation step. Then, we take only recently updated static model parameters and run again the flow simulator from current time $k$ to the next time moment $k$. The dynamic variables obtained replace those got after the measurement update stage of EnKF and become an initial guess for the next time update step. This procedure avoids nonphysical results of the modeling.

The inclusion of the confirmation step into the algorithm results in almost doubling the computational time due to additional forward model run per ensemble member at each time step. In fact, we use the confirming EnKF technique instead of classical ensemble Kalman filtering for our investigations. So, from now on, we mean confirming EnKF technique under the abbreviation of EnKF.

\subsection{History matching via EnKF}

It turns out that EnKF faces an important practical problem, namely, standard deviation of the errors in the state estimate converges very slowly with the number of ensembles. This makes the EnKF quite sensitive to the number of ensemble members used for simulation. Thus, it seems reasonable to start by investigating how filter performance depends on a number of ensemble

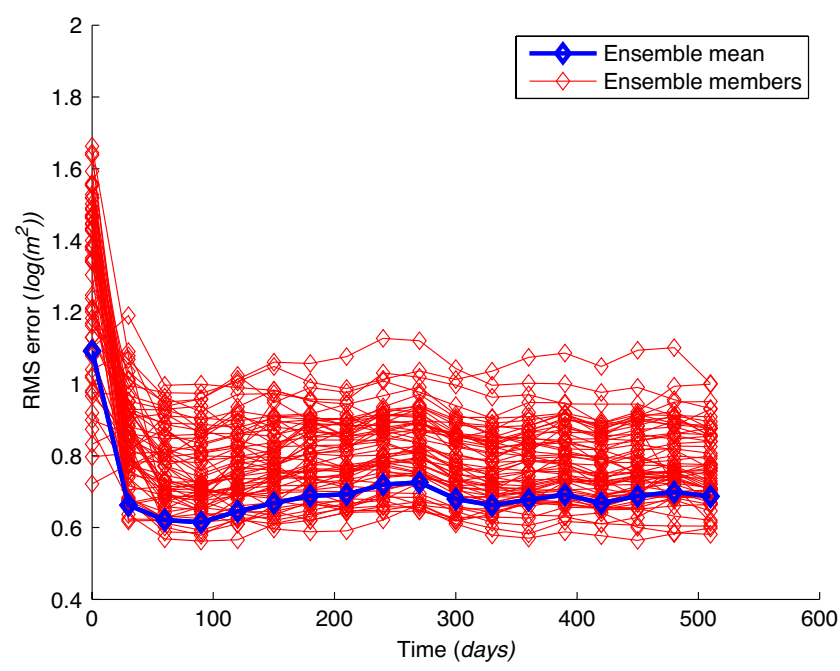

Fig. 4 EnKF: RMS error in estimated permeability vs time

members and find some optimum ensemble size. Preliminary analysis shows that, in our case, performing further EnKF runs on $N=60$ ensemble members seems to be optimal. Since iterative modification of EnKF actually has the same origin, we find it appropriate to use the same ensemble size as being optimal also for IEnKF runs.

Let us now present the outcome of the data assimilation procedure accomplished via the EnKF algorithm with respect to the optimal number of ensemble members. We consider the quality of estimating the model parameter. For that purpose, space-averaged RMS errors Eqs. 13 and 14 are plotted in time (see Fig. 4). These quantities are related to the part of ensemble mean and ensemble members' values corresponding to evaluated permeability. The graph demonstrates improvement of the parameter estimation in the first few data assimilation steps followed by stabilization of the error, and reduction of the uncertainty for estimated value (since the ensemble spread becomes narrower). This means that, at the later times, assimilated data carry less useful information on reservoir structure than at the early times. Indeed, we obtain a permeability field resembling the true one, although some underestimation of the values in the upper right and overestimation of the values in the bottom left corner area of the field occur (compare Fig. 3 and left bottom chart on Fig. 5). The variance field is actually obtained as the diagonal terms of covariance matrix computed from the statistical properties of the ensemble. The difference between the top right and the bottom right subplots in Fig. 5 indicates reduction of the variance and, therefore, uncertainty in the estimation. 


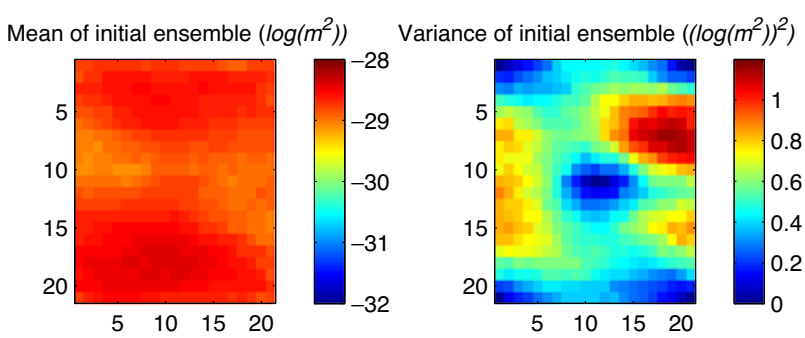

Estimated permeability field $\left(\log \left(m^{2}\right)\right)$ Variance of estimated ensemble $\left(\left(\log \left(m^{2}\right)\right)^{2}\right)$
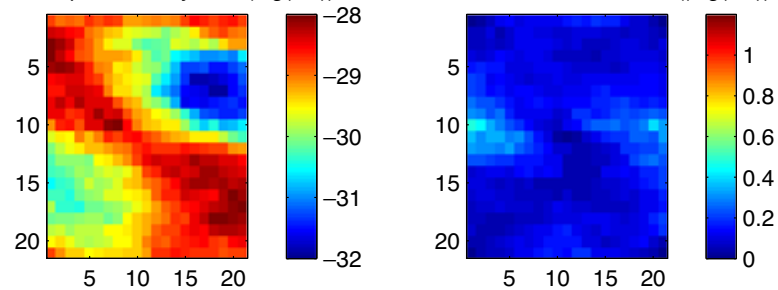

Fig. 5 EnKF: Initial and estimated permeability fields and corresponding variances

Although history matching on the basis of EnKF technique has demonstrated its efficiency for proper estimating model parameters, there is still space for improvement. We may aim at obtaining better representation of reservoir heterogeneous structures, which in turn will result in increasing the quality of forecasts.

\subsection{History matching via IEnKF}

We proceed by running the IEnKF algorithm for the trial example. In fact, we accomplish the second global iteration of the EnKF method. Space averaged RMS errors Eqs. 13 and 14 are plotted in time (see Fig. 6) to evaluate the quality of estimating the model parameter. The graph demonstrates improvement for neither parameter estimation nor uncertainty characterization,

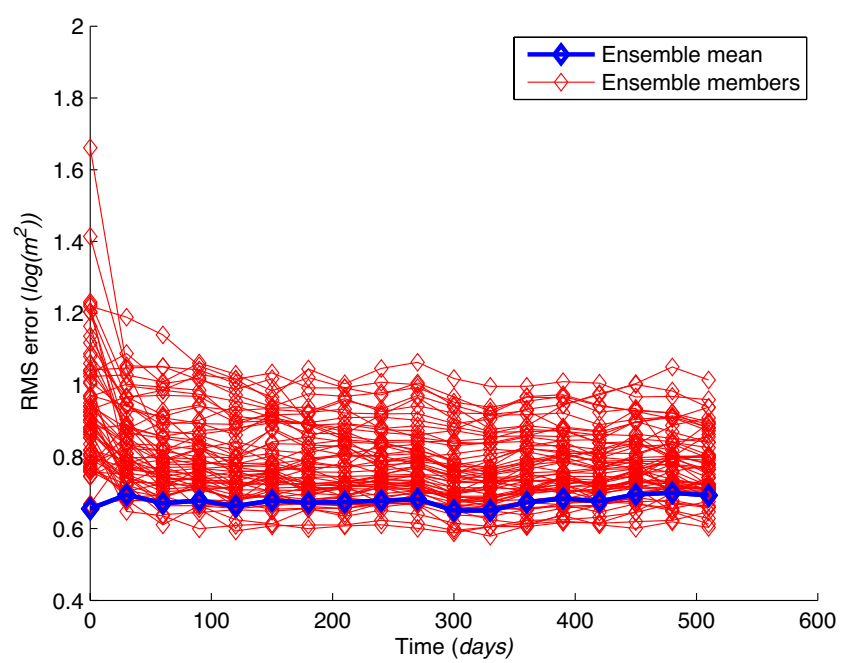

Fig. 6 IEnKF: RMS error in estimated permeability vs time which can be expected since the first EnKF iteration does not provide reducing the parameter estimation error in later times and actually gives us a relatively accurate estimate. Indeed, there is almost no visual difference between permeability fields obtained with EnKF and IEnKF algorithms (compare Figs. 5 and 7).

Consider now a situation when a priori information on the values of model parameters is far from real. For that purpose, we take the initial ensemble of log-permeability fields and shift each member of it by adding the vector $0.5 * \mathbf{I}_{\text {shift }}$, where shifting vector $\mathbf{I}_{\text {shift }}$ consists of ones and $\mathbf{I}_{\text {shift }} \in \mathbf{R}^{1 \times 441}$. Note that such a shift does not affect the variance statistics, hence, the structure of the initial ensemble is kept. The data assimilation is performed from time $t_{0}=0$ days up to time moment $t_{\text {end }}=510$ days, and the covariance of measurement error is scaled by the factor of $10^{2}$ to prevent filter divergence. Such parameters for data assimilation allow some reducing the error in estimation of permeability values performed via IEnKF (see RMS errors Eqs. 13 and 14 plotted in time on Fig. 8). Indeed, we obtain a permeability field with a structure resembling the true one, although some overestimation of the values corresponding to low-permeability areas of the field occurs (see Fig. 9). The parameter values corresponding to these areas are, in particular, improved after global iteration (compare Fig. 9a and b). The difference between the bottom right charts in Fig. 9a and $\mathrm{b}$ indicates reduction of the variance and, therefore, uncertainty in the estimation.

Note that, although regularly providing overestimated values, the filter tends to capture the structure
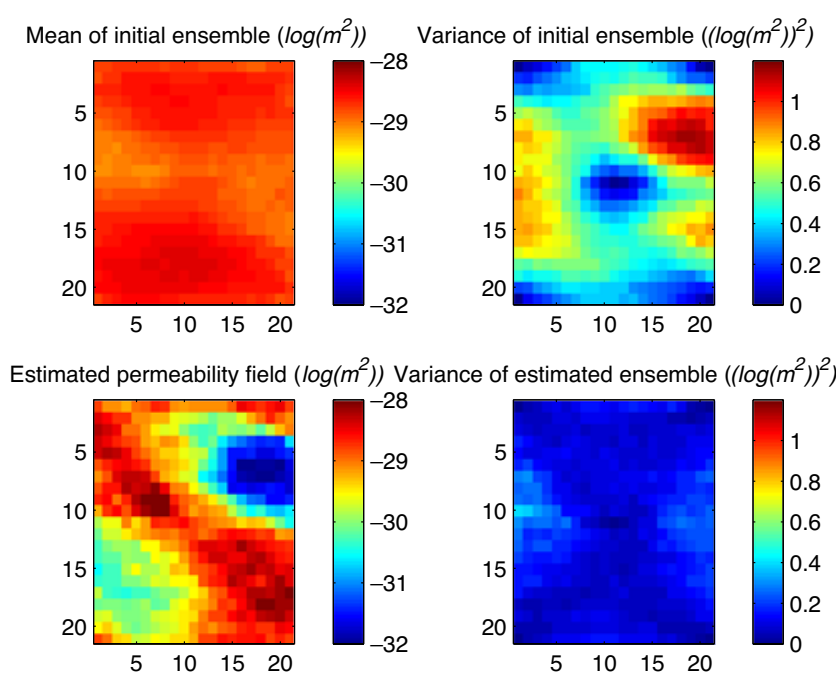

Fig. 7 IEnKF: Initial and estimated permeability fields and corresponding variances 


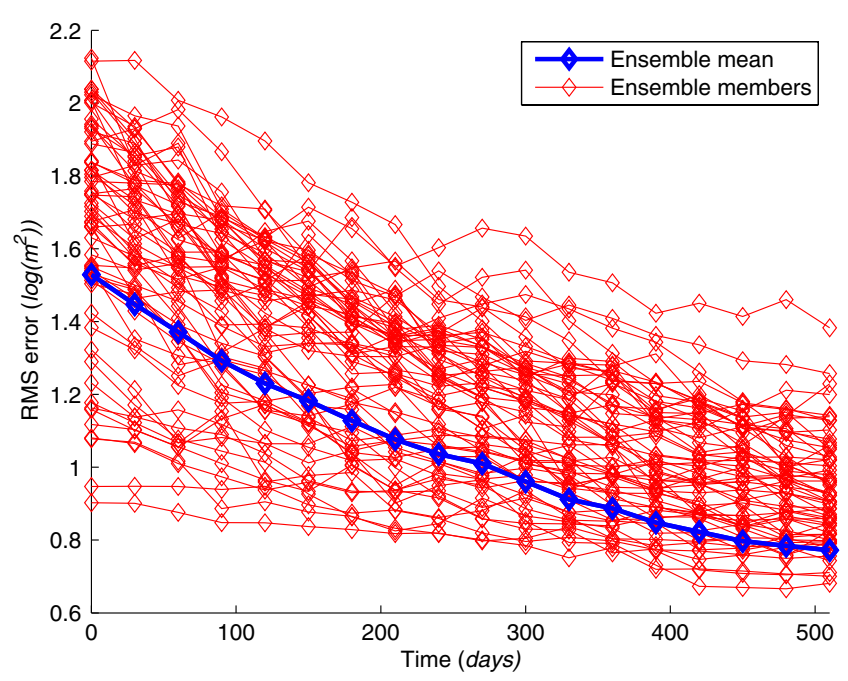

(a)

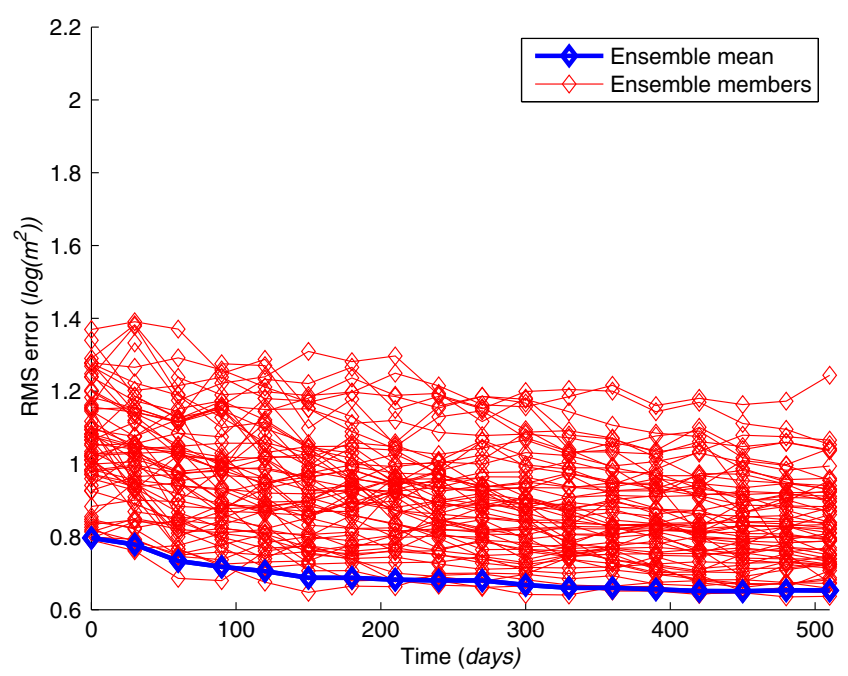

(b)

Fig. 8 IEnKF: RMS error for estimated permeability vs time (shifted initial ensemble with $0.5 * \mathbf{I}_{\text {shift }}$ and measurement error covariance matrix $10^{2} * \mathbf{R}$ are used in experiment). (a) First iteration. (b) Second iteration

of true permeability field. This happens because the ensemble of permeability fields, used for the current test, is only the shifted version of analogous ensemble previously used for investigations. Such an ensemble contains some information on the field structure, which is not influenced by simple shifting since a shift changes the ensemble mean and not the covariance. The given initial statistics cannot be changed because it comes from the statistics of ensemble population. Thus, the possibilities of improving parameter estimation by varying statistics of initially guessed values of model parameter are, in a certain sense, restricted.
Although demonstrating a usage of IEnKF approach for estimating permeability values, the performed tests raise additional problems to be solved. The list of such problems includes finding criteria to evaluate whether global filter iteration is needed in the real case when no true permeability values are available. We suppose that one may consider the RMS differences between the parameter values obtained at two sequential data assimilation steps. Another issue is concerned with determining appropriate error statistics that can have a great impact on improvement of the estimations and the number of global EnKF iterations required for that purpose. Summarizing, we may assert that history
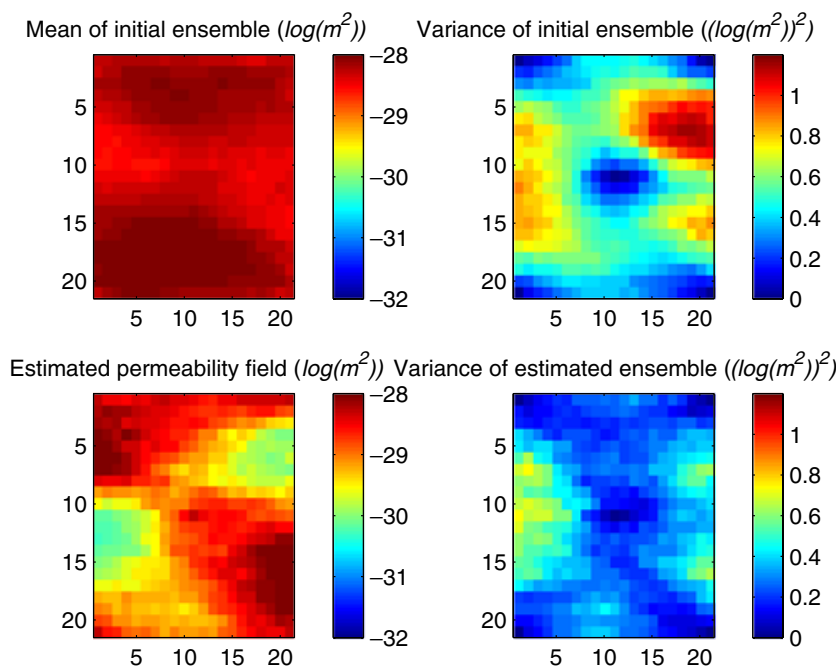

(a)

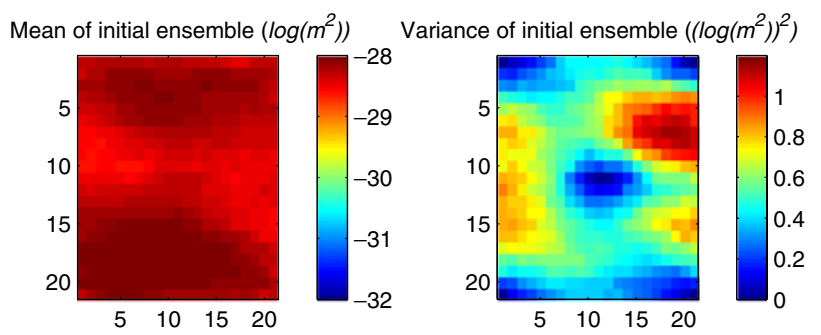

Estimated permeability field $\left(\log \left(m^{2}\right)\right)$ Variance of estimated ensemble $\left(\left(\log \left(m^{2}\right)\right)^{2}\right)$
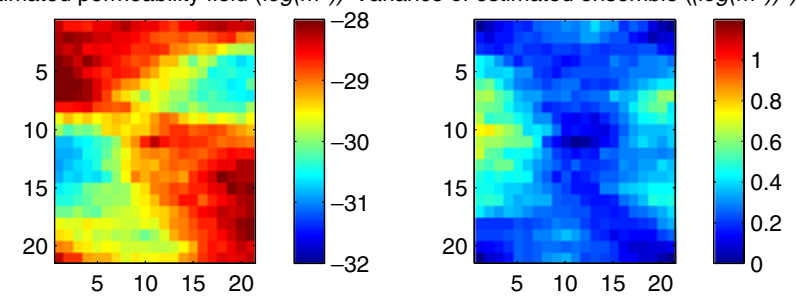

(b)

Fig. 9 IEnKF: Initial and estimated permeability fields and corresponding variances (shifted initial ensemble with $5 * \mathbf{I}_{\text {shift }}$ and measurement error covariance matrix $10^{2} * \mathbf{R}$ are used in experiment). (a) First iteration. (b) Second iteration 
matching on the basis of the IEnKF technique has demonstrated its efficiency for improving model parameter estimation.

\section{Conclusion}

The study has been focused on the analysis of the usage and the applicability of ensemble Kalman filtering techniques with respect to history matching problems. Following the idea presented in [6], an iterative modification of EnKF is proposed. The accomplished case study has confirmed the usefulness of the EnKF technique for solving the history matching problem and estimating reservoir model parameter. There might occur problems at which the EnKF algorithm does not provide results of sufficient accuracy. An appropriate use of the IEnKF method in such a case can improve the estimations.

Acknowledgements The authors would like to thank Prof. J.D. Jansen for providing the reservoir simulator and synthetic reservoir structure. We are grateful to Prof. A.W. Heemink for useful remarks. The comments of the two anonymous reviewers helped to clarify the objective of the paper.

Open Access This article is distributed under the terms of the Creative Commons Attribution Noncommercial License which permits any noncommercial use, distribution, and reproduction in any medium, provided the original author(s) and source are credited.

\section{References}

1. Burgers, G., Leeuwen, P., Evensen, G.: Analysis scheme in the ensemble Kalman filter. Mon. Weather Rev. 126, 17191724 (1998)

2. Ertekin, T., Abou-Kassen, J.H., King, G.R.: Basic Applied Reservoir Simulation. Society of Petroleum Engineers, Richardson (2001)

3. Evensen, G.: The ensemble Kalman filter: theoretical formulation and practical implementation. Ocean Dyn. 53, 343-367 (2003)

4. Gu, Y., Oliver, D.S.: History matching of the PUNQ-S3 reservoir model using the ensemble Kalman filter. In: SPE 89942, SPE Annual Technical Conference and Exhibition, Houston, 26-29 September 2004

5. Gu, Y., Oliver, D.S.: The ensemble Kalman filter for continuous updating of reservoir simulation models. J. Energy Resour. Technol. 128, 79-87 (2006)

6. Jazwinski, A.H.: Stochastic Processes and Filtering Theory. Academic, New York (1970)

7. Lorentzen, R.J., Nævdal, G., Vallès, B., Berg, A.M.: Analysis of the ensemble Kalman filter for estimation of permeability and porosity in reservoir models. In: SPE 96375, SPE Annual Technical Conference and Exhibition, Dallas, 9-12 October 2005

8. Ruijian, L., Reynolds, A.C., Oliver, D.S.: History matching of three-phase flow production data. SPE J. 8(4), 328-340 (2003)

9. Simon, D.: Kalman filtering. Embedded Syst. Program. 14, 72-79 (2001)

10. Wen, X.-H., Chen, W.C.: Real-time reservoir model updating using ensemble Kalman filter. SPE 92991, SPE Reservoir Simulation Symposium, Houston, 31 January-2 February 2005

11. Zafari, M., Reynolds, A.C.: Assessing the uncertainty in reservoir description and performance predicitions with the ensemble Kalman filter. SPE 95750, SPE Annual Technical Conference and Exhibition, Dallas, 9-12 October 2005 